\title{
УДК $\quad 629.783: 527$
}

\section{Устранение смещений взвешенных разностей псевдодальностей, полученных двухчастотным приемником CPHC GPS + ГЛОНАСС}

\author{
В. Е. Вовасов, $\kappa . m$. .., vovasov@list.ru \\ АО «Российские космические системь», Москва, Российская Федерация \\ Д. Н. Чунин, slimdex@mail.ru \\ Министерство оборонь, Москва, Российская Федерация
}

\begin{abstract}
Аннотация. Для устранения смещений оценки псевдодальности, связанной с ионосферой, используют так называемый двухчастотный метод, основанный на формировании взвешенной разности псевдодальностей. Приводятся выражения для взвешенной разности псевдодальностей приемников ГЛОНАСС и GPS для кодов высокой и стандартной точности с учетом аппаратурных задержек как в передающей аппаратуре спутников, так и в навигационных приемниках. Показано, что передаваемой в эфемеридах информации достаточно для нормальной работы приемников GPS и недостаточно для работы приемников ГЛОНАСС. Смещение оценки взвешенной псевдодальности из-за аппаратурных задержек по коду СТ и ВТ требует калибровки приемника ГЛОНАСС. Несмотря на то, что задержка по ВТ-коду между сигналами в диапазонах L1 и L2 передается в эфемеридах, ее точность не удовлетворяет современных потребителей. В связи с этим предлагается производить оценку этого параметра, а также задержек сигналов по ВT- и СТ-кодам в диапазонах L1 и L2 с помощью собственной методики, что обеспечивает учет задержек связанных с аппаратурой передатчиков навигационных спутников. Предложена методика калибровки аппаратурных задержек собственно навигационных приемников с использованием имитатора сигналов GPS + ГЛОНАCC в диапазоне L1 и L2. Показано, что полученная с учетом методик взвешенная псевдодальность для ВТ- и СТ-сигналов ГЛОНАСС имеет смещение, одинаковое для всех литер, и совпадает со смещением взвешенной псевдодальности СТ-сигналов GPS.
\end{abstract}

Ключевые слова: ГЛОНАСС, GPS, псевдодальность, ионосфера, тропосфера

\section{Elimination of Weighted Differences Bias in Pseudoranges Obtained by a Dual-Frequency SRNS Receiver GPS + GLONASS}

\author{
V. E. Vovasov, Cand. Sci. (Engineering),vovasov@list.ru \\ Joint Stock Company "Russian Space Systems", Moscow, Russian Federation \\ D. N.Chunin, slimdex@mail.ru \\ Ministry of Defence, Moscow, Russian Federation
}

\begin{abstract}
To eliminate the pseudorange estimation bias caused by ionosphere influence, the so-called dual-frequency method based on generating weighted difference of pseudoranges is applied. Equations are given for the weighted difference between pseudoranges of GLONASS and GPS receivers for codes of high and standard accuracy taking into account hardware delays both in the transmitting equipment of satellites and in navigation receivers. This method is sufficient for GPS, however is insufficient for normal operation of GLONASS receivers and data transfer via ephemeris. The weighted pseudorange estimation bias due to hardware delays using the standard accuracy (SA) and high accuracy (HA) codes requires GLONASS receiver calibration. Despite the fact that the delay in the HA code between the signals within L1 and L2 bands is transmitted via ephemeris, its accuracy does not satisfy the needs of modern users. Therefore, it is proposed to perform an estimation of this parameter, as well as signal delays in SA and HA codes within L1 and L2 bands, using particular technique, which allows for accounting delays caused by transmitters' equipment of navigation satellites. A technique is proposed for calibrating hardware-related delays particularly for the navigation receivers using the GPS+GLONASS signals simulator within L1 and L2 bands. It is shown that the weighted pseudorange for standard accuracy and high accuracy GLONASS signals, obtained via aforementioned techniques, has the same bias for all types and coincides with the bias of the weighted pseudo-range of the GPS signals with standard accuracy.
\end{abstract}

Keywords: GLONASS, GPS, pseudorange, ionosphere, troposphere 
Для устранения смещений оценки псевдодальности, связанной с ионосферой используют так называемый двухчастотный метод, основанный на формировании взвешенной разности псевдодальностей. Однако этот метод не устраняет смещение оценки псевдодальности, вызванной аппаратурными задержками в самом приемнике. Рассмотрим для начала формирование взвешенной псевдодальности в приемнике GPS.

Выражения для измеряемых в приемнике GPS псевдодальностей по коду высокой точности L1P(Y), L2P(Y) по $j$-му спутнику имеют следующий вид $[5,6]$ :

$$
\begin{gathered}
D_{j, \mathrm{P}(\mathrm{Y})}^{\mathrm{L} 1}\left(t_{i}\right)=R_{j}\left(t_{i}\right)+c \cdot \Delta T_{\mathrm{GPS}}- \\
-c \cdot\left(\Delta T^{j, \mathrm{GPS}}-T_{\mathrm{GD}}^{j}\right)+c\left(T_{\mathrm{trop}}^{j}+T_{\mathrm{ion}, \mathrm{L} 1}^{j}+\tau_{j, \mathrm{~L} 1 \mathrm{P}(\mathrm{Y})}\right)- \\
\quad-\xi_{\mathrm{L} 1 \mathrm{P}(\mathrm{Y})}^{j}, \quad j=\overline{1, J_{1}}, \quad(1) \\
D_{j, \mathrm{P}(\mathrm{Y})}^{\mathrm{L} 2}\left(t_{i}\right)=R_{j}\left(t_{i}\right)+c \cdot \Delta T_{\mathrm{GPS}}- \\
-c \cdot\left(\Delta T^{j, \mathrm{GPS}}-\gamma \cdot T_{\mathrm{GD}}^{j}\right)+c\left(T_{\text {trop }}^{j}+T_{\mathrm{ion}, \mathrm{L} 2}^{j}+\tau_{j, \mathrm{~L} 2 \mathrm{P}(\mathrm{Y})}\right)- \\
-\xi_{\mathrm{L} 2 \mathrm{P}(\mathrm{Y})}^{j}, \quad j=\overline{1, J_{1}}, \quad(2)
\end{gathered}
$$

где $J_{1}$ - количество видимых спутников GPS,

$t_{i}-$ момент формирования измерения. Также введены следующие обозначения:

- $R_{j}$ - длина пути распространения сигнала от фазового центра антенны $j$-го спутника до фазового центра антенны приемника, которая равна

$$
\begin{aligned}
& R_{j}\left(t_{i}\right)= \\
& =\sqrt{\left(x^{j}-x\left(t_{i}\right)\right)^{2}+\left(y^{j}-y\left(t_{i}\right)\right)^{2}+\left(z^{j}-z\left(t_{i}\right)\right)^{2}} .
\end{aligned}
$$

Здесь имеется в виду расстояние между точками, которые занимал $j$-й спутник в момент предшествия и приемник в момент формирования измерения. Под моментом предшествия понимают момент времени, который предшествует моменту формирования измерения на время распространения сигнала;

- $x^{j}, y^{j}, z^{j}-$ координаты $j$-го спутника в момент предшествия, пересчитанные в то положение гринвичской системы координат, которое она занимает в момент измерения псевдодальности;

- $x\left(t_{i}\right), y\left(t_{i}\right), z\left(t_{i}\right)$ - координаты приемника в момент формирования измерения;

- $\Delta T_{\mathrm{GPS}}-$ смещение шкалы времени приемника относительно системной шкалы времени GPS;
- $\Delta T^{j, \mathrm{GPS}}-$ смещение шкалы времени $j$-го спутника относительно шкалы времени GPS;

- $T_{\text {trop }}^{j}-$ задержка кодового сигнала $j$-го спутника в тропосфере;

- $T_{\mathrm{ion}, \mathrm{L} 1}^{j}, T_{\mathrm{ion}, \mathrm{L} 2}^{j}-$ задержка кодового сигнала диапазона L1 и L2 j-го спутника в ионосфере;

- $\tau_{j, \mathrm{~L} 1 \mathrm{P}(\mathrm{Y})}, \tau_{j, \mathrm{~L} 2 \mathrm{P}(\mathrm{Y})}-$ задержка сигнала $\mathrm{L} 1 \mathrm{P}(\mathrm{Y})$ и $\mathrm{L} 2 \mathrm{P}(\mathrm{Y}) j$-го спутника в радиочастотной части приемника;

- $\xi_{\mathrm{L} 1 \mathrm{P}(\mathrm{Y})}^{j}, \xi_{\mathrm{L} 2 \mathrm{P}(\mathrm{Y})}^{j}-$ шумовая составляющая измерения ПД приемником сигнала L1P(Y) и L2P(Y) $j$-го спутника;

- $T_{\mathrm{GD}}^{j}$ - передается в эфемеридах для $j$-го приемника, ее связь с относительной задержкой сигналов L1P(Y) задается в виде

$$
T_{\mathrm{GD}}^{j}=\frac{1}{\gamma-1} \cdot \tau_{\mathrm{L} 2}^{j}
$$

где $\tau_{\mathrm{L} 2}^{j}$ - задержка излучаемого сигнала L2P(Y) $j$-м спутником в радиочастотной части передатчика относительно сигнала L1P(Y).

Для устранения ошибки псевдодальности, связанной с ионосферой, используют так называемый двухчастотный метод, основанный на формировании взвешенной разности псевдодальностей $[5,6]$.

Известна следующая зависимость [1]:

$$
\begin{gathered}
T_{\mathrm{ion}, \mathrm{L} 2}^{j}=\gamma \cdot T_{\mathrm{ion}, \mathrm{L} 1}^{j}, \\
\gamma=\left(\frac{f_{\mathrm{L} 1}^{j}}{f_{\mathrm{L} 2}^{j}}\right)^{2},
\end{gathered}
$$

где $f_{\mathrm{L} 1}^{j}-$ частота несущей сигнала $j$-го спутника в диапазоне L1;

$f_{\mathrm{L} 2}^{j}-$ частота несущей сигнала $j$-го спутника в диапазоне L2.

для GPS

Величина $\gamma=\frac{81}{49}$ для ГЛОНАСС и $\gamma=\left(\frac{77}{60}\right)^{2}$

Умножая выражение (1) на $\gamma$ и вычитая полученное выражение из (2), получим

$$
\begin{gathered}
D_{j, \mathrm{P}(\mathrm{Y})}\left(t_{i}\right)=\frac{D_{j, \mathrm{P}(\mathrm{Y})}^{\mathrm{L} 2}\left(t_{i}\right)-\gamma \cdot D_{j \mathrm{P}(\mathrm{Y})}^{\mathrm{L} 1}\left(t_{i}\right)}{1-\gamma}= \\
=R_{j}\left(t_{i}\right)+c \cdot\left(\Delta T_{\mathrm{GPS}}+\frac{\Delta \tau_{\mathrm{P}(\mathrm{Y})}^{j}}{1-\gamma}\right)-
\end{gathered}
$$




$$
\begin{gathered}
-c \cdot\left(\Delta T^{j, \mathrm{GPS}}\right)+c\left(T_{\text {trop }}^{j}\right)-\frac{\xi_{\mathrm{L} 2 \mathrm{P}(\mathrm{Y})}^{j}-\gamma \cdot \xi_{\mathrm{L} 1 \mathrm{P}(\mathrm{Y})}^{j}}{1-\gamma}= \\
=R_{j}\left(t_{i}\right)+c \cdot\left(\Delta T_{\mathrm{GPS}}+\frac{\Delta \tau_{\mathrm{P}(\mathrm{Y})}}{1-\gamma}\right)- \\
-c \cdot\left(\Delta T^{j, \mathrm{GPS}}\right)+c\left(T_{\text {trop }}^{j}\right)-\frac{\xi_{\mathrm{L} 2 \mathrm{P}(\mathrm{Y})}^{j}-\gamma \cdot \xi_{\mathrm{L} 1 \mathrm{P}(\mathrm{Y})}^{j}}{1-\gamma} \cdot
\end{gathered}
$$

Здесь $\Delta \tau_{\mathrm{P}(\mathrm{Y})}=\Delta \tau_{\mathrm{P}(\mathrm{Y})}^{j}=\tau_{j, \mathrm{~L} 2 \mathrm{P}(\mathrm{Y})}-\gamma \cdot \tau_{j, \mathrm{~L} 1 \mathrm{P}(\mathrm{Y})}-$ величина практически одинаковая для всех принимаемых сигналов, так как сигналы GPS имеют одинаковые полосы и несущие в диапазонах L1 и L2, а значит, и одинаковую задержку в радиочастотной части приемника GPS. Выражения для измеряемых в приемнике GPS псевдодальностей по коду стандартной точности L1 C/A, L2 C по $j$-му спутнику можно представить в виде:

$$
\begin{aligned}
& D_{j, \mathrm{C} / \mathrm{A}}^{\mathrm{L} 1}\left(t_{i}\right)=R_{j}\left(t_{i}\right)+c \cdot \Delta T_{\mathrm{GPS}}- \\
& \quad-c \cdot\left(\Delta T^{j, \mathrm{GPS}}-T_{\mathrm{GD}}^{j}+I S C_{\mathrm{L} 1 \mathrm{C} / \mathrm{A}}^{j}\right)+ \\
& +c\left(T_{\mathrm{trop}}^{j}+T_{\mathrm{ion}, \mathrm{L} 1}^{j}+\tau_{j, L 1 C / A}\right)-\xi_{\mathrm{L} 1 \mathrm{C} / \mathrm{A}}^{j}, \quad j=\overline{1, J_{1}}, \\
& D_{j, \mathrm{C}}^{\mathrm{L} 2}\left(t_{i}\right)=R_{j}\left(t_{i}\right)+c \cdot \Delta T_{\mathrm{GPS}}- \\
& \quad-c \cdot\left(\Delta T^{j, \mathrm{GPS}}-\gamma \cdot T_{\mathrm{GD}}^{j}+\tau_{\mathrm{L} 2}^{j}+I S C_{\mathrm{L} 2 \mathrm{C}}^{j}\right)+ \\
& +c\left(T_{\text {trop }}^{j}+T_{\mathrm{ion}, \mathrm{L} 2}^{j}+\tau_{j, \mathrm{~L} 2 \mathrm{C}}\right)-\xi_{\mathrm{L} 2 \mathrm{C}}^{j}, \quad j=\overline{1, J_{1}},
\end{aligned}
$$

где $I S C_{\mathrm{L} 1 \mathrm{C} / \mathrm{A}}^{j}-$ смещение шкалы времени сигнала L1 P(Y) относительно L1 C/A $j$-го спутника (передается в эфемеридах);

$I S C_{\mathrm{L} 2 \mathrm{C}}^{j}-$ смещение шкалы времени сигнала L1 P(Y) относительно L2 C $j$-го спутника (передается в эфемеридах);

$\tau_{j, \mathrm{~L} 1 \mathrm{C} / \mathrm{A}}, \tau_{j, \mathrm{~L} 2 \mathrm{C}}-$ задержка сигнала L1 C/A и L 2 C $j$-го спутника в радиочастотной части приемника;

$\xi_{\mathrm{L} 1 \mathrm{C} / \mathrm{A}}^{j}, \xi_{\mathrm{L} 2 \mathrm{C}}^{j}-$ шумовая составляющая измерения ПД приемником сигнала L1 C/A и L2 C $j$-го спутника.

Умножая выражение (7) на $\gamma$ и вычитая полученное выражение из (8), получим выражение взвешенной псевдодальности по коду стандартной точности, соответствующее $[5,6]$

$$
\begin{aligned}
& D_{j, \mathrm{C}}\left(t_{i}\right)=\left(D_{j, \mathrm{C}}^{\mathrm{L} 2}\left(t_{i}\right)-\gamma \cdot D_{j, \mathrm{C} / \mathrm{A}}^{\mathrm{L} 1}\left(t_{i}\right)+\right. \\
& \left.+c \cdot\left(I S C_{\mathrm{L} 2 \mathrm{C}}^{j}-\gamma \cdot I S C_{\mathrm{L} 1 \mathrm{C} / \mathrm{A}}^{j}\right)\right) /(1-\gamma)-c \cdot T_{\mathrm{GD}}^{j}=
\end{aligned}
$$

$$
\begin{gathered}
=R_{j}\left(t_{i}\right)+c \cdot\left(\Delta T_{\mathrm{GPS}}+\frac{\Delta \tau_{\mathrm{C}}^{j}}{1-\gamma}\right)-c \cdot\left(\Delta T^{j, \mathrm{GPS}}\right)+ \\
+c\left(T_{\mathrm{trop}}^{j}\right)-\frac{\xi_{\mathrm{L} 2 \mathrm{C}}^{j}-\gamma \cdot \xi_{\mathrm{L} 1 \mathrm{C} / \mathrm{A}}^{j}}{1-\gamma}= \\
=R_{j}\left(t_{i}\right)+c \cdot\left(\Delta T_{\mathrm{GPS}}+\frac{\Delta \tau_{\mathrm{C}}}{1-\gamma}\right)-c \cdot\left(\Delta T^{j, \mathrm{GPS}}\right)+ \\
+c\left(T_{\text {trop }}^{j}\right)-\frac{\xi_{\mathrm{L} 2 \mathrm{C}}^{j}-\gamma \cdot \xi_{\mathrm{L} 1 \mathrm{C} / \mathrm{A}}^{j}}{1-\gamma} .
\end{gathered}
$$

Здесь $\Delta \tau_{\mathrm{C}}=\Delta \tau_{\mathrm{C}}^{j}=\tau_{j, \mathrm{~L} 2 \mathrm{C}}-\gamma \cdot \tau_{j, \mathrm{~L} 1 \mathrm{C} / \mathrm{A}}$ величина практически одинаковая для всех сигналов по той же причине, что и $\Delta \tau_{\mathrm{P}(\mathrm{Y})}^{j}$.

Навигационное решение по $J_{1}$-спутникам позволяет получить оценки координат антенны приемника и величину времени $\Delta T+\frac{\Delta \tau_{\mathrm{P}(\mathrm{Y})}}{1-\gamma}$ при работе по ВТ-сигналам и $\Delta T+\frac{\Delta \tau_{\mathrm{C}}}{1-\gamma}$ при работе по СТ-сигналам. Так как смещение шкалы времени $\frac{\Delta \tau_{\mathrm{P}(\mathrm{Y})}}{1-\gamma}$ или $\frac{\Delta \tau_{\mathrm{C}}}{1-\gamma}$ не превышает $100 \mathrm{Hc}$, то даже для высокоманевренных объектов со скоростями $10000 \mathrm{~m} / \mathrm{c}$ в пересчете на координаты погрешность не превысит $10^{-3}$ м. В связи с этим указанное смещение не учитывается и калибровка приемников GPS не требуется.

Рассмотрим получение аналогичных выражений для приемника ГЛОНАСС.

Приведем известные математические выражения для псевдодальностей по коду высокой точности (ВТ) [2-4]. В ГЛОНАСС в отличие от GPS в качестве бортовой шкалы принята шкала времени сигнала L1BT и именно к ней передаются поправки в эфемеридах. Смещение шкалы времени сигнала L2BT относительно сигнала L1BT обозначим, как и в случае GPS, $\tau_{\mathrm{L} 2, \mathrm{BT}}^{j}$. Эта величина передается в эфемеридах и имеет обозначение $\Delta \tau_{n}^{j}=\tau_{\mathrm{L} 2, \mathrm{BT}}^{j}$. В результате выражения для измеряемых в приемнике псевдодальностей по коду ВТ для двух диапазонов L1 и L2 по $j$-му спутнику имеют следующий вид:

$$
\begin{aligned}
& D_{j, \mathrm{BT}}^{\mathrm{L} 1}\left(t_{i}\right)=R_{j}\left(t_{i}\right)+c \cdot \Delta T-c \cdot\left(\Delta T^{j}\right)+ \\
& +c\left(T_{\text {trop }}^{j}+T_{\mathrm{ion}, \mathrm{L} 1}^{j}+\tau_{j, \mathrm{~L} 1, \mathrm{BT}}\right)-\xi_{\mathrm{L} 1, \mathrm{BT}}^{j} \quad j=\overline{1, J_{2}},
\end{aligned}
$$




$$
\begin{aligned}
& D_{j, \mathrm{BT}}^{\mathrm{L} 2}\left(t_{i}\right)=R_{j}\left(t_{i}\right)+c \cdot \Delta T-c \cdot\left(\Delta T^{j}+\Delta \tau_{n}^{j}\right)+ \\
& +c\left(T_{\text {trop }}^{j}+T_{\mathrm{ion}, \mathrm{L} 2}^{j}+\tau_{j, \mathrm{~L} 2, \mathrm{BT}}\right)-\xi_{\mathrm{L} 2, \mathrm{BT}}^{j}, \quad j=\overline{1, J_{2}},
\end{aligned}
$$

где $J_{2}-$ количество видимых спутников ГЛОНАСС;

$\Delta T$ - смещение шкалы времени приемника относительно системной шкалы времени ГЛОНАСС;

$\Delta T^{j}-$ смещение шкалы времени $j$-го спутника, которая совпадает со шкалой времени сигнала L1BT, относительно системной шкалы времени гЛОНАСС;

$\tau_{j, \mathrm{~L} 1, \mathrm{BT}}, \tau_{j, \mathrm{~L} 2, \mathrm{BT}}-$ задержка кодового ВТ-сигнала диапазона L1 и L2 $j$-го спутника в радиочастотной части приемника;

$\xi_{\mathrm{L} 1, \mathrm{BT}}^{j}, \xi_{\mathrm{L} 2, \mathrm{BT}}^{j}-$ шумовая составляющая измерения ПД приемником по сигналу диапазона L1 и L2 ВT кода $j$-го спутника.

Считается, что все меры для устранения многолучевых искажений приняты и поэтому ошибка, соответствующая этому явлению, здесь не рассматривается.

Умножая выражение (10) на $\gamma$ и вычитая полученное выражение из (11), получим

$$
\begin{aligned}
D_{j, \mathrm{BT}}\left(t_{i}\right)= & \frac{D_{j, \mathrm{BT}}^{\mathrm{L} 2}\left(t_{i}\right)-\gamma \cdot D_{j, \mathrm{BT}}^{\mathrm{L} 1}\left(t_{i}\right)}{1-\gamma}+\frac{c}{1-\gamma} \cdot \Delta \tau_{n}^{j}= \\
= & R_{j}\left(t_{i}\right)+c \cdot \Delta T-c \cdot \Delta T^{j}+c \cdot T_{\mathrm{trop}}^{j}- \\
& -\frac{1}{1-\gamma} \cdot\left(\xi_{\mathrm{L} 2, \mathrm{BT}}^{j}-\gamma \cdot \xi_{\mathrm{L} 1, \mathrm{BT}}^{j}\right)+ \\
& \quad+\frac{c}{1-\gamma} \cdot\left(\tau_{j, \mathrm{~L} 2, \mathrm{BT}}-\gamma \cdot \tau_{j, \mathrm{~L} 1, \mathrm{BT}}\right) . \quad(12)
\end{aligned}
$$

Смещение оценки взвешенной псевдодальности, соответствующей $j$-му спутнику ГЛОНАСС, из-за аппаратурных задержек составляет величину $\frac{c}{1-\gamma} \cdot\left(\tau_{j, \mathrm{~L} 2, \mathrm{BT}}-\gamma \cdot \tau_{j, \mathrm{~L} 1, \mathrm{BT}}\right)$.

Так как сигналы ГЛОНАСС имеют разные несущие частоты, то из-за не идеальности фазовой характеристики усилителя промежуточной частоты величины $\tau_{j, \mathrm{~L} 2, \mathrm{BT}}-\gamma \cdot \tau_{j, \mathrm{~L} 1, \mathrm{BT}}$ не одинаковы для принимаемых сигналов. Разница задержек в радиочастотной части приемника приведены в [7] и составляют $\tau_{j, \mathrm{~L} 2, \mathrm{BT}}-\gamma \cdot \tau_{j, \mathrm{~L} 1, \mathrm{BT}}=20-30$ нс, следовательно, смещение может достигать до 10 м, что вызывает необходимость калибровки.
Приведем известные математические выражения для псевдодальностей по коду стандартной точности (СТ) [2-4]:

$$
\begin{aligned}
& D_{j, \mathrm{CT}}^{\mathrm{L} 1}\left(t_{i}\right)=R_{j}\left(t_{i}\right)+c \cdot \Delta T-c \cdot\left(\Delta T^{j}+\Delta \tau_{\mathrm{L} 1}^{j}\right)+ \\
& +c\left(T_{\text {trop }}^{j}+T_{\text {ion }, \mathrm{L} 1}^{j}+\tau_{j, \mathrm{~L} 1, \mathrm{CT}}\right)-\xi_{\mathrm{L} 1, \mathrm{CT}}^{j}, \quad j=\overline{1, J_{2}}, \\
& D_{j, \mathrm{CT}}^{\mathrm{L} 2}\left(t_{i}\right)=R_{j}\left(t_{i}\right)+c \cdot \Delta T-c \cdot\left(\Delta T^{j}+\Delta \tau_{n}^{j}+\Delta \tau_{\mathrm{L} 2}^{j}\right)+ \\
& +c\left(T_{\text {trop }}^{j}+T_{\text {ion }, \mathrm{L} 2}^{j}+\tau_{j, \mathrm{~L} 2, \mathrm{CT}}\right)-\xi_{\mathrm{L} 2, \mathrm{CT}}^{j}, \quad j=\overline{1, J_{2}},
\end{aligned}
$$

где $\Delta \tau_{\mathrm{L} 1}^{j}=\left(\tau_{\mathrm{L} 1, \mathrm{CT}}^{j}-\tau_{\mathrm{L} 1, \mathrm{BT}}^{j}\right)-$ задержка СТ-кода относительно ВT в диапазоне L1 в аппаратуре спутника;

$\Delta \tau_{\mathrm{L} 2}^{j}=\left(\tau_{\mathrm{L} 2, \mathrm{CT}}^{j}-\tau_{\mathrm{L} 2, \mathrm{BT}}^{j}\right)-$ задержка СТ-кода относительно BT в диапазоне L2 в аппаратуре спутника;

$\tau_{j, \mathrm{~L} 1, \mathrm{CT}}, \tau_{j, \mathrm{~L} 2, \mathrm{CT}}-$ задержка кодового СТ-сигнала диапазона L1 и L2 $j$-го спутника в радиочастотной части приемника;

$\xi_{\mathrm{L} 1, \mathrm{CT}}^{j}, \xi_{\mathrm{L} 2, \mathrm{CT}}^{j}-$ шумовая составляющая измерения псевдодальностей приемником по сигналу диапазона L1 и L2 CT-кода $j$-го спутника.

Умножая выражение (13) на $\gamma$ и вычитая полученное выражение из (14), получим

$$
\begin{aligned}
& D_{j, \mathrm{CT}}\left(t_{i}\right)= \\
& =\frac{D_{j, \mathrm{CT}}^{\mathrm{L} 2}\left(t_{i}\right)-\gamma \cdot D_{j, \mathrm{CT}}^{\mathrm{L} 1}\left(t_{i}\right)+c \cdot\left(\Delta \tau_{\mathrm{L} 2}^{j}-\gamma \cdot \Delta \tau_{\mathrm{L} 1}^{j}\right)}{1-\gamma}+ \\
& \quad+\frac{c}{1-\gamma} \cdot \Delta \tau_{n}^{j}=R_{j}\left(t_{i}\right)+c \cdot \Delta T-c \cdot \Delta T^{j}+ \\
& \quad+c \cdot T_{\text {trop }}^{j}-\frac{1}{1-\gamma} \cdot\left(\xi_{\mathrm{L} 2, \mathrm{CT}}^{j}-\gamma \cdot \xi_{\mathrm{L} 1, \mathrm{CT}}^{j}\right)+ \\
& \quad+\frac{c}{1-\gamma} \cdot\left(\tau_{j, \mathrm{~L} 2, \mathrm{CT}}-\gamma \cdot \tau_{j, \mathrm{~L} 1, \mathrm{CT}}\right) \cdot \quad
\end{aligned}
$$

Смещение оценки взвешенной псевдодальности из-за аппаратурных задержек по коду СТ аналогично смещению по коду ВТ, а значит, требует калибровки приемника ГЛОНАСС. Несмотря на то, что параметр $\Delta \tau_{n}^{j}$ передается в эфемеридах, его точность не удовлетворяет современных потребителей. Учитывая это обстоятельство, до улучшения его точности предлагается производить оценку этого параметра с помощью собственного алгоритма. 
Методика получения задержек

сигналов в аппаратуре передатчиков ГЛОНАСС (величин $\Delta \tau_{n}^{j}, \Delta \tau_{\mathrm{L} 2}^{j}, \Delta \tau_{\mathrm{L} 1}^{j}$ )

Для получения оценок указанных параметров будем использовать сравнение псевдодальностей, полученных при натурных измерениях и с помощью имитатора навигационных сигналов для спутников с одинаковыми номерами. Кроме того, для уменьшения флюктуационной ошибки будем усреднять полученные параметры на интервале $T=$ $=300$ c, так как на этом интервале изменение оцениваемых параметров можно считать несущественным и его величина позволяет получать оцениваемые параметры со среднеквадратической ошибкой не более $0,1 \mathrm{~m}$.

Вычитаем из (10) уравнение (13), запишем

$$
\begin{aligned}
& D_{j, \mathrm{BT}}^{\mathrm{L} 1}\left(t_{i}\right)-D_{j, \mathrm{CT}}^{\mathrm{L} 1}\left(t_{i}\right)= \\
= & c \cdot \Delta \tau_{\mathrm{L} 1}^{j}+c \cdot\left(\tau_{j, \mathrm{~L} 1, \mathrm{BT}}-\tau_{j, \mathrm{~L} 1, \mathrm{CT}}\right)-\xi_{\mathrm{L} 1, \mathrm{BT}}^{j}+\xi_{\mathrm{L} 1, \mathrm{CT}}^{j}
\end{aligned}
$$

Усредняя разности на интервале $T$, получим $\overline{D_{j, \mathrm{BT}}^{\mathrm{L} 1}\left(t_{i}\right)-D_{j, \mathrm{CT}}^{\mathrm{L} 1}\left(t_{i}\right)}=c \cdot \Delta \tau_{\mathrm{L} 1}^{j}+c \cdot\left(\tau_{j, \mathrm{~L} 1, \mathrm{BT}}-\tau_{j, \mathrm{~L} 1, \mathrm{CT}}\right)$.

Вычитаем из (11) уравнение (14), запишем

$$
\begin{aligned}
& D_{j, \mathrm{BT}}^{\mathrm{L} 2}\left(t_{i}\right)-D_{j, \mathrm{CT}}^{\mathrm{L} 2}\left(t_{i}\right)= \\
= & c \cdot \Delta \tau_{\mathrm{L} 2}^{j}+c \cdot\left(\tau_{j, \mathrm{~L} 2, \mathrm{BT}}-\tau_{j, \mathrm{~L} 2, \mathrm{CT}}\right)-\xi_{\mathrm{L} 2, \mathrm{BT}}^{j}+\xi_{\mathrm{L} 2, \mathrm{CT}}^{j} .
\end{aligned}
$$

Усредняя разности на интервале $T$, получим $\overline{D_{j, \mathrm{BT}}^{\mathrm{L} 2}\left(t_{i}\right)-D_{j, \mathrm{CT}}^{\mathrm{L} 2}\left(t_{i}\right)}=c \cdot \Delta \tau_{\mathrm{L} 2}^{j}+c \cdot\left(\tau_{j, \mathrm{~L} 2, \mathrm{BT}}-\tau_{j, \mathrm{~L} 2, \mathrm{CT}}\right)$.

Вычитаем из (11) уравнение (10), запишем

$$
\begin{gathered}
D_{j, \mathrm{BT}}^{\mathrm{L} 2}\left(t_{i}\right)-D_{j, \mathrm{BT}}^{\mathrm{L} 1}\left(t_{i}\right)=-c \cdot \Delta \tau_{n}^{j}+c \cdot\left(T_{\mathrm{ion}, \mathrm{L} 2}^{j}-T_{\mathrm{ion}, \mathrm{L} 1}^{j}\right)+ \\
+c \cdot\left(\tau_{j, \mathrm{~L} 2, \mathrm{BT}}-\tau_{j, \mathrm{~L} 1, \mathrm{BT}}\right)-\xi_{\mathrm{L} 2, \mathrm{BT}}^{j}+\xi_{\mathrm{L} 1, \mathrm{BT}}^{j} .
\end{gathered}
$$

Используя сетку ионосферных вертикальных задержек сигналов GPS или ГЛОНАСС $I_{g}$ сайта -IGS и используя выражение [7]

$$
c \cdot T_{\mathrm{ion}, \mathrm{L} 1}^{j}=I_{g} \cdot \frac{f^{2}}{f_{j, \mathrm{~L} 1}^{2}} \cdot \frac{1}{\sqrt{1-\left[\frac{R_{3}}{R_{3}+h} \cos \left\{\eta_{j}\left(t_{i}\right)\right\}\right]^{2}}},
$$

получим смещение псевдодальности $j$-го сигнала в диапазоне L1, вызванное ионосферой.

Здесь $R_{3}$ - радиус Земли;

$h=432,5 \cdot 10^{3} \mathrm{м}-$ высота слоя ионосферы, где интегральная концентрация электронов в вертикальном столбе достигает $50 \%$;

$f$ - несущая частота, на которой получена оценка $I_{g}$;

$\eta_{j}\left(t_{i}\right)$ - угол места $j$-го навигационного спутника относительно приемника.

Учитывая (4), приведенное выше выражение преобразуем к виду

$$
\begin{array}{r}
D_{j, \mathrm{BT}}^{\mathrm{L} 2}\left(t_{i}\right)-D_{j, \mathrm{BT}}^{\mathrm{L} 1}\left(t_{i}\right)+c \cdot\left(T_{\mathrm{ion}, \mathrm{L} 1}^{j}\right) \cdot(1-\gamma)= \\
=-c \cdot \Delta \tau_{n}^{j}\left(t_{i}\right)+c \cdot\left(\tau_{j, \mathrm{~L} 2, \mathrm{BT}}-\tau_{j, \mathrm{~L} 1, \mathrm{BT}}\right)- \\
-\xi_{\mathrm{L} 2, \mathrm{BT}}^{j}+\xi_{\mathrm{L} 1, \mathrm{BT}}^{j}
\end{array}
$$

Усредняя на интервале $T$, получим

$$
\begin{array}{r}
\overline{D_{j, \mathrm{BT}}^{\mathrm{L} 2}\left(t_{i}\right)-D_{j, \mathrm{BT}}^{\mathrm{L} 1}\left(t_{i}\right)+c \cdot\left(T_{\mathrm{ion}, \mathrm{L} 1}^{j}\right) \cdot(1-\gamma)}= \\
=-c \cdot \Delta \tau_{n}^{j}\left(t_{i}\right)+c \cdot\left(\tau_{j, \mathrm{~L} 2, \mathrm{BT}}-\tau_{j, \mathrm{~L} 1, \mathrm{BT}}\right) .
\end{array}
$$

Очевидно, что выражения (16)-(18) содержат неизвестные величины, представляющие разности аппаратурных задержек в навигационном приемнике ГЛОНАСС. Для их получения используем имитатор, имеющий выход СТ- и ВТ-кода в диапазоне L1 и L2. Сценарий должен быть написан таким образом, что $T_{\text {trop }}^{j}=0, T_{\text {ion, } \mathrm{L} 1}^{j}=0, c \cdot\left(\Delta T^{j}\right)=0$, $\Delta R^{j}=0, \Delta \tau_{n}^{j}=0, \Delta \tau_{\mathrm{L} 1}^{j}=0, \Delta \tau_{\mathrm{L} 2}^{j}=0$.

Тогда измерения псевдодальностей при использовании имитатора могут быть представлены в виде

$$
\begin{aligned}
D_{j, \mathrm{BT}}^{\mathrm{L} 1}\left(t_{i}\right) & =R_{j}\left(t_{i}\right)+c \cdot \Delta T+ \\
& +c \cdot \tau_{j, \mathrm{~L} 1, \mathrm{BT}}-\xi_{\mathrm{L} 1, \mathrm{BT}}^{j}, \quad j=\overline{1, J}, \\
D_{j, \mathrm{BT}}^{\mathrm{L} 2}\left(t_{i}\right) & =R_{j}\left(t_{i}\right)+c \cdot \Delta T+ \\
& +c \cdot \tau_{j, \mathrm{~L} 2, \mathrm{BT}}-\xi_{\mathrm{L} 2, \mathrm{BT}}^{j}, \quad j=\overline{1, J}, \\
D_{j, \mathrm{CT}}^{\mathrm{L} 1}\left(t_{i}\right) & =R_{j}\left(t_{i}\right)+c \cdot \Delta T+ \\
& +c \cdot \tau_{j, \mathrm{~L} 1, \mathrm{CT}}-\xi_{\mathrm{L} 1, \mathrm{CT}}^{j}, \quad j=\overline{1, J}, \\
D_{j, \mathrm{CT}}^{\mathrm{L} 2}\left(t_{i}\right) & =R_{j}\left(t_{i}\right)+c \cdot \Delta T+ \\
& +c \cdot \tau_{j, \mathrm{~L} 2, \mathrm{CT}}-\xi_{\mathrm{L} 2, \mathrm{CT}}^{j}, \quad j=\overline{1, J} .
\end{aligned}
$$

РАКЕТНО-КОСМИЧЕСКОЕ ПРИБОРОСТРОЕНИЕ И ИНФОРМАЦИОННЫЕ СИСТЕМЫ т. 4 ВЫП. 42017 
Вычитая из (19) уравнение (21), получим

$$
\begin{aligned}
& D_{j, \mathrm{BT}}^{\mathrm{L} 1}\left(t_{i}\right)-D_{j, \mathrm{CT}}^{\mathrm{L} 1}\left(t_{i}\right)= \\
& \quad=c \cdot\left(\tau_{j, \mathrm{~L} 1, \mathrm{BT}}-\tau_{j, \mathrm{~L} 1, \mathrm{CT}}\right)-\xi_{\mathrm{L} 1, \mathrm{BT}}^{j}+\xi_{\mathrm{L} 1, \mathrm{CT}}^{j} .
\end{aligned}
$$

Усредняем разности на интервале $T$, запишем

$$
\begin{aligned}
& C_{j}=\bar{D}_{j, \mathrm{BT}}^{\mathrm{L} 1}\left(t_{i}\right)-D_{j, \mathrm{CT}}^{\mathrm{L} 1}\left(t_{i}\right) \text { имитатор } \\
&=c \cdot\left(\tau_{j, \mathrm{~L} 1, \mathrm{BT}}-\tau_{j, \mathrm{~L} 1, \mathrm{CT}}\right) .
\end{aligned}
$$

Вычитая из (16) выражение (23), получим

$$
\begin{aligned}
& \overline{D_{j, \mathrm{BT}}^{\mathrm{L} 1}\left(t_{i}\right)-D_{j, \mathrm{CT}}^{\mathrm{L} 1}\left(t_{i}\right)}- \\
& \quad-\overline{D_{j, \mathrm{BT}}^{\mathrm{L} 1}\left(t_{i}\right)-D_{j, \mathrm{CT}}^{\mathrm{L} 1}\left(t_{i}\right)}{ }^{\text {имитатор }}=c \cdot \Delta \tau_{\mathrm{L} 1}^{j} .
\end{aligned}
$$

Вычитая из (20) уравнение (22), получим

$$
\begin{aligned}
D_{j, \mathrm{BT}}^{\mathrm{L} 2}\left(t_{i}\right) & -D_{j, \mathrm{CT}}^{\mathrm{L} 2}\left(t_{i}\right)= \\
& =c \cdot\left(\tau_{j, \mathrm{~L} 2, \mathrm{BT}}-\tau_{j, \mathrm{~L} 2, \mathrm{CT}}\right)-\xi_{\mathrm{L} 2, \mathrm{BT}}^{j}+\xi_{\mathrm{L} 2, \mathrm{CT}}^{j} .
\end{aligned}
$$

Усредняем разности на интервале $T$, запишем

$$
\begin{aligned}
V_{j}={\overline{D_{j, \mathrm{BT}}^{\mathrm{L} 2}\left(t_{i}\right)-D_{j, \mathrm{CT}}^{\mathrm{L} 2}\left(t_{i}\right)}}^{=c \cdot\left(\tau_{j, \mathrm{~L} 2, \mathrm{BT}}-\tau_{j, \mathrm{~L} 2, \mathrm{CT}}\right) .} & \\
& =
\end{aligned}
$$

Вычитая из (17) выражение (24), получим

$$
\begin{aligned}
& \overline{D_{j, \mathrm{BT}}^{\mathrm{L} 2}\left(t_{i}\right)-D_{j, \mathrm{CT}}^{\mathrm{L} 2}\left(t_{i}\right)}- \\
& \quad-\overline{D_{j, \mathrm{BT}}^{\mathrm{L} 2}\left(t_{i}\right)-D_{j, \mathrm{CT}}^{\mathrm{L} 2}\left(t_{i}\right)}{ }^{\text {имитатор }}=c \cdot \Delta \tau_{\mathrm{L} 2}^{j} .
\end{aligned}
$$

Вычитая из (20) выражение (19), получим

$$
\begin{aligned}
& D_{j, \mathrm{BT}}^{\mathrm{L} 2}(\left.t_{i}\right)-D_{j, \mathrm{BT}}^{\mathrm{L} 1}\left(t_{i}\right)= \\
& \quad=c \cdot\left(\tau_{j, \mathrm{~L} 2, \mathrm{BT}}-\tau_{j, \mathrm{~L} 1, \mathrm{BT}}\right)-\xi_{\mathrm{L} 2, \mathrm{BT}}^{j}+\xi_{\mathrm{L} 1, \mathrm{BT}}^{j} .
\end{aligned}
$$

Усредняем на интервале $T$, запишем

$$
\begin{aligned}
Z_{j}={\overline{D_{j, \mathrm{BT}}^{\mathrm{L} 2}\left(t_{i}\right)-D_{j, \mathrm{BT}}^{\mathrm{L} 1}\left(t_{i}\right)}}^{=c}= \\
=c\left(\tau_{j, \mathrm{~L} 2, \mathrm{BT}}-\tau_{j, \mathrm{~L} 1, \mathrm{BT}}\right) .
\end{aligned}
$$

Подставляя в (18) выражение (25), получим

$$
\begin{aligned}
c & \cdot \Delta \tau_{n}^{j}\left(t_{i}\right)=\overline{D_{j, \mathrm{BT}}^{\mathrm{L} 2}\left(t_{i}\right)-D_{j, \mathrm{BT}}^{\mathrm{L} 1}\left(t_{i}\right)}{ }^{\mathrm{Lмитатор}}- \\
& -\overline{D_{j, \mathrm{BT}}^{\mathrm{L} 2}\left(t_{i}\right)-D_{j, \mathrm{BT}}^{\mathrm{L} 1}\left(t_{i}\right)+c \cdot\left(T_{\mathrm{ion}, \mathrm{L} 1}^{j}\right) \cdot(1-\gamma)} .
\end{aligned}
$$

Очевидно, что для получения $\Delta \tau_{n}^{j}, \Delta \tau_{\mathrm{L} 2}^{j}, \Delta \tau_{\mathrm{L} 1}^{j}$ необходимы как натурные измерения навигационного приемника в диапазоне L1 и L2 по коду CT и ВТ, так и измерения полученные с помощью имитатора. Если в эфемеридах величина $\Delta \tau_{n}^{j}$ будет передаваться с погрешностью менее $0,1 \mathrm{~m}$, то проведение измерений (18) и (25), а значит, и привлечение данных сайта -IGS не потребуется.

Использование выражения (12) приводит к необходимости раздельной оценки $c \cdot \tau_{j, \mathrm{~L} 1, \mathrm{BT}}$ и $c \times$ $\times \tau_{j, \mathrm{~L} 2 \text {,Вт }}$, а использование выражения (15) приводит к необходимости раздельной оценки $c \cdot \tau_{j, \mathrm{~L} 1, \mathrm{CT}}$ и $c \cdot \tau_{j, \mathrm{~L} 2, \mathrm{CT}} \cdot$ Так как выражение $c \cdot\left(\tau_{j, \mathrm{~L} 2, \mathrm{CT}}-\right.$ $\left.-\tau_{j, \mathrm{~L} 1, \mathrm{CT}}\right)$ является линейной комбинацией трех вышеприведенных выражений (23), (24) и (25), то получение раздельной оценки требуемых задержек без дополнительных измерений не представляется возможным.

Так как в нашем случае для обработки сигналов используется приемник GPS + ГЛОНАСС, то для решения указанной проблемы предлагается воспользоваться постоянством групповой задержки сигналов в приемнике GPS по коду СТ $\Delta \tau_{\mathrm{C}}$ и получить зависимости задержек $\tau_{j, \mathrm{~L} 1, \mathrm{BT}}, \tau_{j, \mathrm{~L} 2, \mathrm{BT}}, \tau_{j, \mathrm{~L} 1, \mathrm{CT}}$, $\tau_{j, \mathrm{~L} 2, \mathrm{CT}}$ от этой величины.

\section{Калибровка приемника (получение}

$$
\begin{aligned}
& c \cdot \tau_{j, \mathrm{~L} 1, \mathrm{BT}}, c \cdot \tau_{j, \mathrm{~L} 2, \mathrm{BT}}, c \cdot \tau_{j, \mathrm{~L} 1, \mathrm{CT}} \\
& \text { и } c \cdot \tau_{j, \mathrm{~L} 2, \mathrm{CT}} \text { для всех литер) }
\end{aligned}
$$

Используем имитатор GPS + ГЛОНАСС сигналов в диапазоне L1 и L2. Сценарий должен быть написан таким образом, чтобы для GPS-сигналов выполнялись следующие условия $T_{\text {trop }}^{j}=0$, $T_{\text {ion } \mathrm{L} 1}^{j}=0, c \cdot\left(\Delta T^{j}\right)=0, \Delta R^{j}=0, T_{\mathrm{GD}}=$ $=0, I S C_{\mathrm{L} 1 \mathrm{C} / \mathrm{A}}=0, I S C_{\mathrm{L} 2 \mathrm{C}}=0$, а для сигналов ГЛОНАСС $-\Delta \tau_{n}^{j}=0, \Delta \tau_{\mathrm{L} 1}^{j}=0, \Delta \tau_{\mathrm{L} 2}^{j}=0$. Кроме этого, задано совпадение шкал времени системы GPS и ГЛОНАСС.

Тогда измерения псевдодальностей по сигналам ГЛОНАСС и GPS могут быть представлены в виде

$$
\begin{aligned}
D_{j, \mathrm{C} / \mathrm{A}}^{\mathrm{L} 1}\left(t_{i}\right) & =R_{j}\left(t_{i}\right)+c \cdot \Delta T+ \\
& +c \cdot\left(\tau_{j, \mathrm{~L} 1 \mathrm{C} / \mathrm{A}}\right)-\xi_{\mathrm{L} 1 \mathrm{C} / \mathrm{A}}^{j}, \quad j=\overline{1, J_{1}}, \\
D_{j, \mathrm{C}}^{\mathrm{L} 2}\left(t_{i}\right) & =R_{j}\left(t_{i}\right)+c \cdot \Delta T+ \\
& +c \cdot\left(\tau_{j, \mathrm{~L} 2 \mathrm{C}}\right)-\xi_{\mathrm{L} 2 \mathrm{C}}^{j}, \quad j=\overline{1, J_{1}},
\end{aligned}
$$




$$
\begin{aligned}
D_{k, \mathrm{CT}}^{\mathrm{L} 1}\left(t_{i}\right) & =R_{k}\left(t_{i}\right)+c \cdot \Delta T+ \\
& +c \cdot\left(\tau_{k, \mathrm{~L} 1, \mathrm{CT}}\right)-\xi_{\mathrm{L} 1, \mathrm{CT}}^{k}, \quad k=\overline{1, J_{2}}, \\
D_{k, \mathrm{CT}}^{\mathrm{L} 2}\left(t_{i}\right) & =R_{k}\left(t_{i}\right)+c \cdot \Delta T+ \\
& +c \cdot\left(\tau_{k, \mathrm{~L} 2, \mathrm{CT}}\right)-\xi_{\mathrm{L} 2, \mathrm{CT}}^{k}, \quad k=\overline{1, J_{2}} .
\end{aligned}
$$

Номер $k$ для ГЛОНАСС выбран потому, чтобы не было путаницы при совпадении номеров спутников различных систем.

Вычитаем из (28) выражение (26), получим

$$
\begin{aligned}
c \cdot\left(\tau_{k, \mathrm{~L} 1, \mathrm{CT}}\right)- & \cdot\left(\tau_{j, \mathrm{~L} 1 \mathrm{C} / \mathrm{A}}\right)=D_{k, \mathrm{CT}}^{\mathrm{L} 1}\left(t_{i}\right)-D_{j, \mathrm{C} / \mathrm{A}}^{\mathrm{L} 1}\left(t_{i}\right)- \\
- & R_{k}\left(t_{i}\right)+R_{j}\left(t_{i}\right)-\xi_{\mathrm{L} 1, \mathrm{CT}}^{k}+\xi_{\mathrm{L} 1 \mathrm{C} / \mathrm{A}}^{j}
\end{aligned}
$$

Усредняем за $T$, получим

$$
\begin{aligned}
& A_{k}= \\
& ={\overline{D_{k, \mathrm{CT}}^{\mathrm{L} 1}\left(t_{i}\right)-D_{j, \mathrm{C} / \mathrm{A}}^{\mathrm{L} 1}\left(t_{i}\right)-R_{k}\left(t_{i}\right)+R_{j}\left(t_{i}\right)}}^{\text {имитатор }}= \\
& =c \cdot\left(\tau_{k, \mathrm{~L} 1, \mathrm{CT}}\right)-c \cdot\left(\tau_{j, \mathrm{~L} 1 \mathrm{C} / \mathrm{A}}\right) \text {. }
\end{aligned}
$$

Вычитаем из (29) выражение (27), получим

$$
\begin{array}{r}
c \cdot\left(\tau_{k, \mathrm{~L} 2, \mathrm{CT}}\right)-c \cdot\left(\tau_{j, \mathrm{~L} 2 \mathrm{C}}\right)=D_{k, \mathrm{CT}}^{\mathrm{L} 2}\left(t_{i}\right)-D_{j, \mathrm{C}}^{\mathrm{L} 2}\left(t_{i}\right)- \\
-R_{k}\left(t_{i}\right)+R_{j}\left(t_{i}\right)-\xi_{\mathrm{L} 2, \mathrm{CT}}^{k}+\xi_{\mathrm{L} 2 \mathrm{C}}^{j}
\end{array}
$$

Усредняем за $T$, получим

$$
\begin{aligned}
& B_{k}= \\
& ={\overline{D_{k, \mathrm{CT}}^{\mathrm{L} 2}\left(t_{i}\right)-D_{j, \mathrm{C}}^{\mathrm{L} 2}\left(t_{i}\right)-R_{k}\left(t_{i}\right)+R_{j}\left(t_{i}\right)}}^{\text {имитатор }}= \\
& =c \cdot\left(\tau_{k, \mathrm{~L} 2, \mathrm{CT}}\right)-c \cdot\left(\tau_{j, \mathrm{~L} 2 \mathrm{C}}\right) \text {. }
\end{aligned}
$$

Умножая (30) на $\gamma$ и вычитая из (31), получим для каждой $k$-й литеры

$$
\begin{array}{r}
c \cdot\left(\tau_{k, \mathrm{~L} 2, \mathrm{CT}}-\gamma \cdot \tau_{k, \mathrm{~L} 1, \mathrm{CT}}\right)-c \cdot\left(\tau_{j, \mathrm{~L} 2 \mathrm{C}}-\gamma \cdot \tau_{j, \mathrm{~L} 1 \mathrm{C} / \mathrm{A}}\right)= \\
=B_{k}-\gamma \cdot A_{k} .
\end{array}
$$

\section{Отсюда}

$c \cdot\left(\tau_{k, \mathrm{~L} 2, \mathrm{CT}}-\gamma \cdot \tau_{k, \mathrm{~L} 1, \mathrm{CT}}\right)=B_{k}-\gamma \cdot A_{k}+c \cdot \Delta \tau_{\mathrm{C}}$.

Подставляя (32) в выражение (12), получим

$$
\begin{aligned}
& D_{k, \mathrm{CT}}^{\prime}\left(t_{i}\right)=D_{k, \mathrm{CT}}\left(t_{i}\right)-\frac{1}{1-\gamma} \cdot\left(B_{k}-\gamma \cdot A_{k}\right)= \\
= & \frac{D_{k, \mathrm{CT}}^{\mathrm{L} 2}\left(t_{i}\right)-\gamma \cdot D_{k, \mathrm{CT}}^{\mathrm{L} 1}\left(t_{i}\right)+c \cdot\left(\Delta \tau_{\mathrm{L} 2}^{k}-\gamma \cdot \Delta \tau_{\mathrm{L} 1}^{k}\right)}{1-\gamma}+
\end{aligned}
$$

$$
\begin{gathered}
+\frac{c}{1-\gamma} \cdot \Delta \tau_{n}^{k}-\frac{1}{1-\gamma} \cdot\left(B_{k}-\gamma \cdot A_{k}\right)= \\
=R_{k}\left(t_{i}\right)+c \cdot\left(\Delta T+\frac{\Delta \tau_{\mathrm{C}}}{1-\gamma}\right)-c \cdot \Delta T^{k}+c \cdot T_{\text {trop }}^{k}- \\
\quad-\frac{1}{1-\gamma} \cdot\left(\xi_{\mathrm{L} 2, \mathrm{CT}}^{k}-\gamma \cdot \xi_{\mathrm{L} 1, \mathrm{CT}}^{k}\right) \cdot
\end{gathered}
$$

Очевидно, что полученная таким образом взвешенная псевдодальность для СТ-сигналов ГЛОНАСС имеет смещение одинаковое для всех литер и совпадает со смещением взвешенной псевдодальности СТ-сигналов GPS. Определим взвешенную псевдодальность для всех литер ВТ-сигналов ГЛОНАСС.

Используя выражения (23), (24), (25), получим

$$
c \cdot \tau_{k, \mathrm{~L} 2, \mathrm{CT}}-c \cdot \tau_{k, \mathrm{~L} 1, \mathrm{CT}}=C_{k}-V_{k}+Z_{k} .
$$

Отсюда

$c \cdot \tau_{k, \mathrm{~L} 1, \mathrm{CT}}=\frac{B_{k}-\gamma \cdot A_{k}+c \cdot \Delta \tau_{\mathrm{C}}-C_{k}+V_{k}-Z_{k}}{1-\gamma}$,

$$
\begin{aligned}
& c \cdot \tau_{k, \mathrm{~L} 2, \mathrm{CT}}=c \cdot \tau_{k, \mathrm{~L} 1, \mathrm{CT}}+C_{k}-V_{k}+Z_{k}= \\
& =\frac{B_{k}-\gamma \cdot A_{k}+c \cdot \Delta \tau_{\mathrm{C}}-\gamma \cdot\left(C_{k}+V_{k}-Z_{k}\right)}{1-\gamma}, \\
& c \cdot \tau_{k, \mathrm{~L} 1, \mathrm{BT}}=C_{k}+c \cdot \tau_{k, \mathrm{~L} 1, \mathrm{CT}} \\
& =\frac{B_{k}-\gamma \cdot A_{k}+c \cdot \Delta \tau_{\mathrm{C}}-\gamma \cdot C_{k}+V_{k}-Z_{k}}{1-\gamma}, \\
& c \cdot \tau_{k, \mathrm{~L} 2, \mathrm{BT}}=V_{k}+c \cdot \tau_{k, \mathrm{~L} 2, \mathrm{CT}}= \\
& =V_{k}+\frac{B_{k}-\gamma \cdot A_{k}+c \cdot \Delta \tau_{\mathrm{C}}-\gamma \cdot\left(C_{k}+V_{k}-Z_{k}\right)}{1-\gamma} .
\end{aligned}
$$

Используя выражения (37) и (36), запишем равенство

$$
\begin{aligned}
c \cdot\left(\tau_{k, \mathrm{~L} 2, \mathrm{BT}}-\gamma \cdot \tau_{k, \mathrm{~L} 1, \mathrm{BT}}\right) & = \\
=V_{k}+B_{k}-\gamma \cdot A_{k} & +c \cdot \Delta \tau_{\mathrm{C}}-\gamma \cdot C_{k}- \\
& -\frac{2 \cdot \gamma}{1-\gamma} \cdot\left(V_{k}-Z_{k}\right) .
\end{aligned}
$$

Перепишем выражение (12) с учетом (38) и получим

$$
\begin{aligned}
& D_{k, \mathrm{BT}}^{\prime}\left(t_{i}\right)=D_{k, \mathrm{BT}}\left(t_{i}\right)- \\
& -\frac{V_{k}+B_{k}-\gamma \cdot A_{k}-\gamma \cdot C_{k}-\frac{2 \cdot \gamma}{1-\gamma} \cdot\left(V_{k}-Z_{k}\right)}{1-\gamma}=
\end{aligned}
$$




$$
\begin{aligned}
= & \frac{D_{k, \mathrm{BT}}^{\mathrm{L} 2}\left(t_{i}\right)-\gamma \cdot D_{k, \mathrm{BT}}^{\mathrm{L} 1}\left(t_{i}\right)}{1-\gamma}+\frac{c}{1-\gamma} \cdot \Delta \tau_{n}^{k}- \\
- & \frac{V_{k}+B_{k}-\gamma \cdot A_{k}-\gamma \cdot C_{k}-\frac{2 \cdot \gamma}{1-\gamma} \cdot\left(V_{k}-Z_{k}\right)}{1-\gamma}= \\
= & R_{k}\left(t_{i}\right)+c \cdot\left(\Delta T+\frac{\Delta \tau_{\mathrm{C}}}{1-\gamma}\right)-c \cdot \Delta T^{k}+ \\
& +c \cdot T_{\text {trop }}^{k}-\frac{1}{1-\gamma} \cdot\left(\xi_{\mathrm{L} 2, \mathrm{BT}}^{k}-\gamma \cdot \xi_{\mathrm{L} 1, \mathrm{BT}}^{k}\right) .
\end{aligned}
$$

Очевидно, что полученная таким образом взвешенная псевдодальность для ВТ-сигналов ГЛОНАСС имеет смещение, одинаковое для всех литер, и совпадает со смещением взвешенной псевдодальности СТ-сигналов GPS. Приведенный подход позволяет без дополнительных погрешностей решать навигационную задачу приемника GPS + + ГЛОНАСС как совместно по СТ- и ВТ-сигналам, так и раздельно, а также получать навигационное решение как по сигналам ГЛОНАСС, так и по сигналам GPS как совместно, так и раздельно.

\section{Выводы}

1. Выражение (6) взвешенной разности псевдодальностей для GPS по ВТ-коду требует знания величины $T_{\mathrm{GD}}^{j}$ для каждого спутника (передается в эфемеридах). Выражение (9) взвешенной разности псевдодальностей для GPS по СТ-коду требует знания дополнительно величин $I S C_{\mathrm{L} 2 \mathrm{C}}^{j}, I S C_{\mathrm{L} 1 \mathrm{C} / \mathrm{A}}^{j}$ для каждого спутника (передаются в эфемеридах). Выражение (12) взвешенной разности псевдодальностей для ГЛОНАСС по ВТ-коду требует знания величины $\Delta \tau_{n}^{j}$ для каждого спутника (передается в эфемеридах с низкой точностью). Выражение (15) взвешенной разности псевдодальностей для ГЛОНАСС по СТ-коду дополнительно требует знания величин $\Delta \tau_{\mathrm{L} 2}^{j}, \Delta \tau_{\mathrm{L} 1}^{j}$ для каждого спутника (в эфемеридах не предусмотрены).

2. Величины $\Delta \tau_{n}^{j}, \Delta \tau_{\mathrm{L} 2}^{j}, \Delta \tau_{\mathrm{L} 1}^{j}$, относящиеся к работе навигационных спутников ГЛОНАСС, должны вычисляться в НКУ ГЛОНАСС, передаваться на борт каждого спутника и быть включены в эфемериды. В настоящий момент эти величины можно получать с помощью приведенной в работе методики.

3. В выражениях взвешенной разности псевдодальностей (6), (9), (12) и (15), предназначенных для устранения смещения оценки псевдодальности по коду стандартной и высокой точности, связанной с ионосферой, для двухчастотного приемника GPS + ГЛОНАСС присутствуют параметры, связанные с аппаратурными задержками в самом приемнике, приводящие к смещению оценки взвешенной псевдодальности.

4. Приведенная в работе калибровка позволяет привести смещение оценки взвешенной псевдодальности для сигналов ГЛОНАСС по СТ-коду в выражении (33) и по ВТ-коду в выражении (39) до постоянной величины по каждой литере, равной смещению псевдодальности для сигналов GPS по СТ-коду. Такой подход позволяет получить практически не смещенные оценки координат и смещение шкалы времени приемника относительно системы на величину, равную $\Delta \tau_{\mathrm{C}} /(1-\gamma)$ для сигналов как ГЛОНАСС, так и GPS.

\section{Список литературы}

1. ГЛОНАСС. Принципы построения и функционирования / Под ред. А. И. Перова, В. Н. Харисова. Изд. 4-е, перераб. М: Радиотехника, 2010. 800 с.

2. Поваляев A.A. Спутниковые радионавигационные системы: время, показания часов, формирование измерений и определение относительных координат. M.: Радиотехника, 2008. 328 с.

3. Поваляев А. А., Вейцель В. А., Мазепа Р. Б. Глобальные спутниковые системы синхронизации и управления в околоземном пространстве: Учеб. пособие / Под ред. А. А. Поваляева. М.: Вузовская книга, 2012. $188 \mathrm{c}$.

4. ГЛОНАСС. Интерфейсный контрольный документ. Навигационный радиосигнал в диапазонах L1, L2 (редакция 5.1). Российский научно-исследовательский институт космического приборостроения (2008). Проверено 21 октября 2016.

5. IS-GPS-200D, Navstar GPS Space Segment/Navigation User Interfaces, 7 March, 2006.

6. IS-GPS-200F, Navstar GPS Space Segment/Navigation User Interfaces, 21-SEP-2011.

7. Вовасов В.Е., Ипкаев Н.Б. Методика определения аппаратурных задержек сигнала для двухчастотного приемника СРНС ГЛОНАСС // Ракетно-космическое приборостроение и информационные системы, 2014, т. 1, вып. 2. С. 25-32. 


\section{References}

1. GLONASS. Printsipy postroeniya $i$ funktsionirovaniya [GLONASS. Principles of design and operation]. Ed. by Perov A. I., Kharisov V.N. 4th Ed., revised. Moscow, Radiotekhnika, 2010, 800 p. (in Russian)

2. Povalyaev A. A. Sputnikovye radionavigatsionnye sistemy: vremya, pokazaniya chasov, formirovanie izmereniy $i$ opredelenie otnositel'nykh koordinat [Satellite radio navigation systems: time, clock readings, formation of measurements and determination of relative coordinates]. Moscow, Radiotekhnika, 2008, 328 p. (in Russia)

3. Povalyaev A. A., Veytsel' V. A., Mazepa R. B. Global'nye sputnikovye sistemy sinkhronizatsii $i$ upravleniya v okolozemnom prostranstve: ucheb. Posobie [Global satellite systems for synchronization and control in near-Earth space: Textbook]. Ed. by A. A. Povalyaev. Moscow, Vuzovskaya kniga, 2012, 188 p. (in Russian)
4. GLONASS. Interfeysnyy kontrol'nyy dokument. Navigatsionnyy radiosignal v diapazonakh L1, L2 [GLONASS. Interface control document. Navigation radio signal in the L1, L2 bands] Edition 5.1). Rossiyskiy nauchno-issledovatel'skiy institut kosmicheskogo priborostroeniya [Russian Scientific Research Institute of Space Instrument Engineering] (2008). Acessed October 21, 2016. (in Russian)

5. IS-GPS-200D, Navstar GPS Space Segment/Navigation User Interfaces, 7 March, 2006.

6. IS-GPS-200F, Navstar GPS Space Segment/Navigation User Interfaces, 21 September, 2011.

7. Vovasov V. E., Ipkaev N. B. Metodika opredeleniya apparaturnykh zaderzhek signala dlya dvukhchastotnogo priemnika SRNS GLONASS [Technique of Definition of Hardware Delays of a Signal for Two-Frequency Receiver SRNS GLONASS]. Raketno-kosmicheskoe priborostroenie $i$ informatsionnye sistemy [Rocket-Space Device Engineering and Information Systems]. 2014, Vol. 1, No 2, pp. 25-32. (in Russian) 